\title{
Sur l'introduction expérimentale du premier signe humain dans les paysages naturels
}

\author{
Sobre la experimentación del primer indicio humano \\ en los paisages naturales
}

\section{About experience of first human signs in natural landscapes}

\author{
Jean Paul Forest \\ Artist, B.P.3264, Papeete, 98713 Tahiti, Polynésie Française \\ E-mail: jeanpaulforest@mail.pf
}

\begin{abstract}
Resumé
Mes interventions artistiques ont été suscitées par la rupture de statut d'un espace mythologique personnel et sa domestication. D’abord réactionnel, mon travail consista à recoudre les failles de rochers sur des lieux destinés à être industrialisés, afin de réaliser un lien ultime avec l'éphémère, la beauté et le mythe. Puis l'extension technologique m'a conduit à rechercher des sites extrêmement isolés, où l'oeuvre est un échange personnel avec le lieu qui la suggère, et auquel elle est destinée. Ces traces alimentèrent de nouvelles légendes, déclenchèrent des polémiques et trouvèrent l'appui des pouvoirs publics. Les photographies de ces paysages couturés sont exposées à un public ignorant les réalités locales afin de le confronter à l'image de nos activités dans un espace et un temps sans limites. Les sites vierges idéalisent l'idée de nature sans réveiller notre peur. La trace humaine y est donc ambiguë : souillure ou parure, complicité ou agression ? Ainsi évolue notre conception de la nature, au fil du modelage réciproque entre paysages et humanité.
\end{abstract}

Mots clés: couture, domestication, nature, paysages, Papenoo

\section{Resumen}

Mis intervenciones artisticas han sido suscitadas por la ruptura del estatuto de un espacio mitológico personal y de su domesticación. Primeramente reaccional, mi trabajo consistió en coser las fisuras de rocas situadas en lugares destinados a ser « industrializados » con el fin de realizar una última analogía entre lo efímero, la belleza y el mito. Posteriormente, la extensión tecnológica me ha conducido a buscar parajes extremadamente aislados, dónde la obra es un intercambio personal con el lugar que la sugiere y al cual está destinada. Estos indicios alimentaron nuevas leyendas, desencadenaron la polémica y encontraron el apoyo de los poderes públicos. Las fotografías de estos paisages « recosidos » son expuestos a un público ignorante de las realidades locales, con la finalidad de confrontarlos a la imagen de nuestras actividades dentro de un espacio y de un tiempo ilimitado. Los parajes vírgenes idealizan la idea de naturaleza sin despertar nuestro miedo. La huella humana es, por tanto, ambigua: ensuciar o decorar, complicidad o agresión ? Así evoluciona nuestra concepción de la naturaleza, mientras se amoldan recíprocamente paisages y humanidad.

Palabras-clave: costura, domesticación, naturaleza, paisages, Papenoo. 


\section{Abstract}

My artistic work has been susceptible by the status crash of a personal mystic environment and his domestication. First of all reactional, my work consisted to sew faults of rocks in places to become industrialised, in order to make a last link between ephemeral, beauty and myth. Later, technological expansion has lead me to go in search of very isolated sites, where my work is a personal exchange whit the site who suggest it, and which it is destined. This traces supplied new legends, launched controversial and found support of public government. Pictures of those sewed landscapes are showed to a public unaware of the local reality, so that it will be confronted to the view of our activities into a living space with no limits. Virgin sites idealise nature without wake up our fear. So the human trace is ambiguous: sully or finery, complicity or aggression ? This way evolve our conception of nature, while reciprocally shaping landscape and humanity.

Key-words: to sew, domestication, nature, landscapes, Papenoo.

\section{La relation à un lieu}

Entre l'œuvre pariétale et son milieu naturel sont tissés des liens cruciaux et significatifs. Les dimensions géographique, architecturale mais surtout imaginaire du site sont essentielles tant pour l'auteur des œuvres que pour ses spectateurs. Ainsi à mon arrivée à Tahiti en 1979, j’ai eu le privilège de parcourir un site exceptionnel. Au milieu du plus vaste océan de la planète, une île produite par un volcan s'érode et s'enfonce lentement dans l'océan, avec au centre le cratère effondré qui retient les nuages. Les eaux de pluie s’écoulent vers la mer dans une vallée taillée par des dizaines de torrents : la vallée de Papenoo.

A l'arrivée des Européens, elle abritait une importante population vivant de la culture du taro sur des terrasses aménagées aux confluents des rivières, de l'élevage de porcs, et du façonnement d'herminettes exceptionnelles échangées dans tout l'archipel de la Société. Cette vallée, du fait de sa situation centrale, était un lieu d'échanges commerciaux, de négociations guerrières, de neutralité pour les vaincus, et de cultes sur d’innombrables édifices de pierre, les marae. Les missionnaires chrétiens cherchèrent à regrouper les Polynésiens sur les côtes pour mieux les contrôler ; c'est donc à Papenoo que se réfugièrent les réfractaires, et que subsista jusqu’à la fin du dixneuvième siècle un mode de vie et des cultes de l'époque néolithique (1). La vallée ne fut plus ensuite parcourue que par des pécheurs et des chasseurs de cochons sauvages. De vagues sentiers longeaient parfois des marae en ruine recouverts par la végétation. 
Au milieu du vingtième siècle quelques expéditions archéologiques fouillèrent les sites les plus accessibles. La vallée était alors considérée comme un espace immense, labyrinthique et mystérieux. Le souvenir d'une culture pré-européenne l’imprégnait : croyances en des pirogues fantômes descendant la rivière la nuit, d’âmes errantes des ancêtres, de tabou frappant d'anciens lieux de culte et des sépultures dont les emplacements étaient oubliés. C’était une porte vers les temps anciens et un espace insondable.

Puis brusquement en 1986 un aménagement hydroélectrique de la haute vallée bouleversa tout. Une piste fut tracée, des sauvetages archéologiques réalisés dans la précipitation. Ce basculement du mythe à la réalité domestique suscita mes premières interventions : le passage d'un "ordre naturel" à une ère technologique m’a interrogé sur ce qui pousse l’humanité à s'élaborer et se définir essentiellement à travers ses actions sur la matière du monde. En temps que plasticien, j'ai donc exploré de manière concrète cette rupture introduite par le premier signe humain.

\section{La confrontation à l'éphémère}

La transformation des paysages par des engins de terrassement, l'édification de barrages, d’usines ou décharges à ordure, matérialisaient le changement de statut. Ma première phase d'interventions fut réactionnelle au sentiment de profanation de ce lieu dont je m’étais construit une image idéalisée. Deux motifs m’ont conduit à agir :

- le désir de passer de longs moments dans des sites provisoirement préservés, de réaliser physiquement un lien avec l'éphémère, la beauté et le mythe : un adieu précédant une agonie annoncée

- la volonté ironique d'aller à contre courant d’une énorme vague industrielle.

D'un point de vue pratique, j'ai sélectionné des sites exprimant, du moins en apparence, un état facilement identifiable : puissance et omniprésence d'une nature vierge. Pour y exprimer une première trace matérielle humaine, j’ai choisi de réduire au 1/1000 ème les actions qui se déroulaient autour de moi : on posait des kilomètres de tuyaux de 3 mètres de diamètre, j'allais sur quelques mètres poser des câbles de 3 millimètres. J'ai donc recousu les failles naturelles de rochers avant le passage des bulldozers, coupé méticuleusement des pierres pour les suturer ensuite. Ces interventions se situent à proximité des zones de passage, voire sur des aires totalement 
détruites par la suite. Ces coutures dérisoires en périphérie des transformations industrielles sont à la fois les réparations pathétiques d'un lieu défiguré et la matérialisation d'une limite, comme on renforce un tissu en voie de dégradation. On y retrouve les symboliques de signalisation pour marquer une frontière, et d'offrande pour réparer un tort causé. Mais apparaît aussi la fermeture par la force, comme la “ bouche cousue " marque l'impossibilité de parler donc de se défendre, comme la suture rituelle du sexe féminin est une soumission. Ainsi toute couture reste ambiguë : tout en étant une réparation elle participe à la dégradation, ou du moins en est l'image miniaturisée et méticuleuse. Souillure ou parure, caresse ou blessure, complicité ou agression ? Ces deux pôles opposés induisent une question cruciale : assumons-nous l’image de nos propres actions sur le monde, et selon quels critères ?

\section{L'exploration du mythe}

L'extension progressive de l'emprise technologique sur toute la longueur de la vallée m’a conduit à une seconde phase d'interventions en des lieux plus isolés. Le constat que tout site considéré comme vierge, voire mythique, est susceptible de tomber un jour dans le domaine domestique m’a libéré de la nécessité d’un sens social à mes actes. Le ciel lui-même, universellement lieu de séjour des divinités, étant désormais parcouru par nos avions et nos satellites, nous en modifions irréversiblement la perception y compris pour les rares groupes humains n'ayant pas de contact avec la civilisation occidentale. C'est l'exemple typique d'annexion ipso facto d'espaces sacrés collectifs à des fins utilitaires.

Créer un lien avec un lieu devint donc une affaire privée, où ne sont concernés que le site qui m'attire et ma propre conscience, puisqu'aucun espace n'est par essence à l'abri d’une utilisation fonctionnelle : une trace laissée en un lieu vierge apparaîtra-t-elle comme un sacrilège ou une sacralisation ? J’ai alors tenté de produire des œuvres induites uniquement par et pour le paysage, telles des broderies. Ce travail typiquement féminin est réalisé avec des outils et matériaux contemporains et masculins, mais sur une matière symboliquement féminine. La vallée de Papenoo se présente en effet comme une arborescence de torrents, entre des parois la plus part du temps verticales, formant de par la végétation tropicale et les innombrables cascades 
barrant les cours d'eau un vaste espace labyrinthique, univers infini dans un espace fini. C’est un immense corps qui s’offre au ciel et aux humains.

Les efforts en temps et en difficulté pour rejoindre le lieu de l'œuvre agissent comme un préparateur, un épurateur. Ils m’extirpent mentalement du monde domestique : c’est un voyage initiatique préparant à la rencontre avec le site et la réalisation d'un lien privilégié. Le cheminement fait partie de l'œuvre, et un accès laborieux donne d'une part la sensation de se retirer hors de l'emprise sociale, d'autre part d'aller à la rencontre de la matière du monde dans ce qu'elle a de plus intime (avec sa complicité puisque le labyrinthe s’est laissé déchiffrer), et par conséquent d’avancer vers la partie la plus authentique de soi-même.

Le temps consacré au site est hors du temps profane, hors d'un regard ou jugement culturel ou social. Le lieu est une entité que l’on vient rencontrer, avec lequel on dialogue et choisi pour la sensation particulière qu'il procure. De chacun émane une ambiance et une harmonie différente qui se reflètent en nous, provoquant une attraction et des réactions spécifiques. Les lieux ayant un “ esprit ” très marqué ont toujours attiré les hommes selon des critères essentiellement subjectifs certes, mais parfaitement reproductibles au fil du temps, des individus et des cultures. Il convient de s’interroger sur les différentes raisons nous ayant poussé à les marquer de notre empreinte, alors que par essence ces sites se suffisent à eux-mêmes pour générer leur mana. Désir de se soumettre à leur pouvoir, d’y participer, d'en récupérer un bénéfice, ou d'en vaincre la puissance ?

La trace laissée (par moi-même sur la matière et réciproquement) intègre à la fois le trajet, le séjour, et le matériau modifié. Elle est l'expression du lien ressenti (ou imaginé), l'artiste étant à la fois venu chercher et déposer. L'oeuvre est livrée aux forces naturelles qui la conserveront, la modifieront et l'effaceront. Elle est une interface entre l'univers et l'humain, mais aussi entre le conscient et l'inconscient de l'artiste. Elle est un échange personnel avec la matière qui la supporte, et à laquelle elle est destinée (2). 


\section{Implications sociales et culturelles}

Réalisées sans témoin ni information du public, certaines coutures furent découvertes par des guides de randonnée, qui ont formulé des explications logiques pour leur clientèle:

- système pour consolider des orgues basaltiques, ou pour en mesurer le mouvement

- œuvre d’un américain vivant dans la montagne (légende d’hommes occidentaux redevenus “ sauvages ” vivant à l'écart de la civilisation) que l’on ne voit jamais et qui coud les pierres pour des raisons mystérieuses.

Découvertes par des Polynésiens pécheurs ou chasseurs, ceux-ci y voyaient des traces laissées par leurs ancêtres, sans tenir compte de l'utilisation de matériaux contemporains. Lorsque leur nature réelle fut connue, quelques sentiers ont été tracés pour en faciliter l'accès. Les “ coureurs des bois ” qui parcourent la vallée ont perçu le défi ironique à l'avancée des travaux de transformation de la vallée, et les associations de protection de Papenoo les ont adoptées.

Ultérieurement, leur présentation hors contexte, par photographies ou vidéo dans des expositions, a visé à confronter un public ignorant les réalités locales avec la transgression fondatrice de ce travail. La transposition en image à la fois découpe une fenêtre dans un paysage qu'elle magnifie et idéalise, et montre la couture, seule trace humaine, comme sujet de document. Les réactions favorables semblent suscitées par une analogie entre cette trace manufacturée perdue dans un espace vierge et une perception de la condition humaine. Le geste réalisé affirme notre existence dans ce monde, et y revendique une place. Ces images replacent l'aventure humaine dans des dimensions de temps et d'espace sans limites, et donc sans valeur extrinsèque : tout est question de trajectoire, d'angle de vue, de distance, d'éclairage. Un point de vue esthétique suggère un équilibre possible, inscription poétique jetée à travers l'espace et le temps.

Les réactions défavorables sont essentiellement liées au sentiment d’une agression irréparable et impardonnable : une souillure défigure sans raison un paysage considéré comme parfait. Dénuées de rôle domestique et n’étant pas l'expression d'un élan collectif qui les justifieraient, ces coutures révoltent parce qu'elles vont à l'encontre de l'idée usuelle de protection absolue que l'on devrait à la virginité de la nature. L’illusion d'un mode de vie technologique, confortable et urbain mais propre, 
c'est à dire n’empiétant pas sur des espaces préservés, semble être une condition nécessaire à la bonne conscience collective. Si l'extension de la maîtrise technologique est le fil conducteur de la culture qui s’impose sur la planète, l’image de cet événement semble cependant inacceptable par une grande partie des individus qui s'y impliquent spontanément. Quel reflet acceptons-nous d'avoir de nous même, en temps qu'espèce biologique et que sujets culturels ? Il convient de se pencher sur notre manière de considérer le paysage, et nos interactions avec lui.

\section{De la réalité à une image du paysage}

Si le terme " paysage " désigne usuellement un ensemble visuel, il est cependant fait de la somme des informations sensorielles que nous délivrent nos cinq sens. Mais seules les informations que notre culture nous a apprises à décoder seront retenues, et deux individus de la même culture, selon leurs histoires personnelles, seront sensibles de manière variable voir opposée aux mêmes stimuli. De plus, chaque site sollicite une somme d'informations pratiques : la connaissance ou l'ignorance des dangers et des ressources d'un lieu modifient notablement la manière de laquelle il se projette en nous. Se superpose également tout un imaginaire, conscient ou non, fait de connaissances historiques, de croyances religieuses, de superstitions populaires, d'angoisses ou de mythes personnels. L'impact d'un paysage sur un individu est plus la révélation d'un espace intérieur que l'espace extérieur lui-même.

Le paysage représenté photographiquement se réduisant à la seule perception visuelle, la dimension subjective de son interprétation est fortement amplifiée. Ne rentrent plus en compte les modes d'accès au site, les conditions de vie, l'environnement climatique, biologique ou sonore. Dans le cas du paysage dit naturel, le spectateur est essentiellement projeté vers une interprétation idéalisée de la nature, considérée comme la référence des origines, le zéro de l'action humaine, la pureté et la stabilité mythique disparues des sites recomposés par l'homme. Cette perception idyllique est pourtant en contradiction avec ce que nous révèlent les comportements et fantasmes collectifs vis-à-vis de celle-ci. Des singes géants aux extra-terrestres, des microbes mortels aux météorites tombés du ciel, de la vengeance d'esprits courroucés aux bouleversements climatiques, chaque époque exprime à sa manière la phobie de périls venus des espaces qu'elle ne contrôle pas, c'est-à-dire du vrai domaine naturel. 
Dans ce contexte paradoxal, la trace humaine est elle-même ambiguë. Hors critique si elle est le fruit d'un élan collectif ou religieux, source de fierté si elle représente un exploit technique ou monumental, rassurante si elle correspond à une fonction domestique, elle est ipso facto acceptée et intégrée au paysage, participant même à son esthétique (3). Mais le cumule de ces interventions, et surtout les moyens techniques de mise en œuvre qu'elles nécessitent, vont périodiquement faire craindre la rupture d'un ordre préexistant à l'humain, la coupure fatidique d'avec la matière qui nous a produits. Si l'extension de notre emprise sur des territoires nouveaux naît en partie de notre crainte de la nature, elle va la refouler mais la raviver en même temps, via la culpabilité de la transgression et la nostalgie des édens ancestraux. D'où la nécessité de sanctuaires, de zones autonomes de référence, dans lesquelles les actions humaines seraient bannies.

Mais le sanctuaire, pour être actif culturellement, doit être accessible. Ainsi se poursuit le paradoxe : même une réserve naturelle devra être gérée et se couvrira d'équipements, d'accès facilités, de pancartes et poubelles. En prétendant offrir la nature au public (et un public à la nature), on tisse un réseau domestique ce qui permet d'en réduire la "sauvagerie", et la menace inconsciente qu'elle représente (4). D’une part ce n’est plus le même paysage que l'on offre à voir puisqu'il est parsemé de signes humains, et surtout un accès transformé ne peut donner un impact identique sur les individus. La fréquentation de la vallée de Papenoo par de rares chasseurs et pécheurs il y a 25 ans, l'absence de voie d'accès, les mythologies de sites sacrés enfouis dans la végétation, d’âmes errantes des ancêtres et de pirogues fantômes descendant la rivière la nuit en faisait un lieu magique pour qui s’y aventurait. Traverser aujourd'hui les mêmes sites dans une file de 4X4 climatisés diffusant de la musique, peut-il encore donner la même impression, sous prétexte que rien n’a changé lorsqu’on lève les yeux vers les sommets ...

Nos préoccupations économiques et techniques étant les fils conducteurs de notre culture, elles sont la justification ipso facto de l'utilisation de tout espace accessible comme matière première domestique. Premiers réceptacles des forces motrices des sociétés, les paysages sont donc en devenir constant comme elles. Chaque modification crée d'autres références culturelles, elles-mêmes générant des individus différents. Ainsi émerge en permanence une nouvelle humanité, ayant d’autres capacités 
et d'autres exigences, induites cette fois-ci par la culture bien plus rapidement que la biologie ne pourrait le faire (5). Cette autre humanité à son tour modèle son environnement selon ses propres critères, créant ainsi la rupture avec ses prédécesseurs devenus inadaptés, et préparant celle avec ses successeurs qui grandiront dans un milieu inédit. Paysages et humanité sont liés dans un processus évolutif du à un modelage réciproque, et sans doute incontrôlable.

\section{Confrontation entre deux états de la nature}

Cette expérimentation de la rupture induite par la trace humaine m'a conduit à exposer ces photographies d'une nature puissante et sauvage comportant un premier et infime signe humain, dans les jardins du Musée d'Art Moderne et d'Art Contemporain de Liège, reste de nature totalement domestiquée et noyée dans un monde exclusivement urbain. Il y a pourtant entre les deux une continuité car il fut bien un temps où, à la place de cette ville, il n'y eut que des pierres, de l'eau, des plantes et des animaux ; puis deux cent mille ans au moins avant notre ère, un premier campement de chasseurs pré néandertaliens (6).

Depuis cette époque où l'homme essayait de se glisser dans les jeux d'une nature changeante, nous avons beaucoup évolués. Les paysages portent les marques significatives, comme autant de symboles, des valeurs conscientes et inconscientes des peuples les ayant façonnés. Comme le regard que nous portons sur eux, le comportement que nous avons face aux paysages est lui aussi grandement conditionné par nos valeurs culturelles, qui ne nous suggèrent qu'un éventail limité d'attitudes. Par exemple, certains Aborigènes australiens apprenaient à leurs enfants à ne pas laisser traîner leur lance au sol, afin de ne pas marquer inutilement la terre. Celle-ci est considérée comme une entité à ne pas désorganiser, et la vie comme la contemplation d'un espace où l'homme n'est qu'un invité de passage (7). La notion de Paysage prend là toute sa dimension : l'humain est face au monde, il doit s'y glisser grâce à une connaissance complexe, et suivre le mouvement de ce grand corps sur lequel il n'est que spectateur éphémère et privilégié.

Lassés de nous adapter, ou déjà captivés par notre ingéniosité, nous avons tenté d'adapter le milieu à nos besoins. Avec les civilisations de l'agriculture puis de l’industrie, tout est pensé en terme d'aménagements, la terre étant devenue un réservoir 
de matières à disposition des besoins et de l'imagination de l'humanité. Cela implique l'extension de notre emprise sur des territoires nouveaux et notre modification permanente des espaces déjà conquis, phénomène si constant depuis des millénaires en Occident qu'il apparaît comme la norme. Finalement, il est devenu nécessaire à chaque individu de marquer de sa trace propre le lieu de son existence. Il nous faut des tâches à accomplir, celles-ci étant nécessaires à la fois à remplir le temps de notre vie, et à exprimer que nous existons en temps qu'individu. Cela nous conduit à affirmer notre personnalité et notre pouvoir sur le paysage à notre portée : un chef d'état la planète, un maire sa ville, une femme au foyer son intérieur. Notre mode de vie est basé sur une activité effrénée et toujours renouvelée, créant une ivresse supposée masquer le questionnement métaphysique inhérent à la condition humaine.

\section{Perspectives}

Si l'opposition nature/culture ou nature/humain est depuis longtemps fortement controversée d'un point de vue philosophique et anthropologique, nous continuons cependant en pratique d'appliquer ce regard distinctif. Notre jugement sur le monde, nos actes et notre évolution se réfèrent à cette grille de lecture. La place de ce que nous appelons " nature " s'est fortement renforcée ces dernières décennies, à travers les inquiétudes écologiques et les quêtes identitaires. L'idée de nature, péjorative il n'y a pas si longtemps par ce qu'elle comporte d'incontrôlé et donc dangereux, est devenue positive en réaction à la pression exponentielle exercée par les activités humaines. Mais ce concept de nature a lui même fortement évolué. Pour une population maintenant essentiellement urbaine, que représente l'idée de nature ? Celle dans laquelle l'homme occidental rêve " de retourner " est inoffensive et confortable. Aménagée depuis des millénaires en Europe, en voie d'aménagement là où le tourisme se développe, nous parlons en fait d'une verdure de plus en plus jardinée, accessible, sécurisée. La nature " sauvage " continue donc de régresser, à la fois dans nos têtes et dans la réalité du monde. La photographie d'un paysage totalement vierge d'action humaine est l'image d'un mythe, là où personne n'a plus vraiment l'opportunité de s'aventurer. Ainsi les espaces naturels restent les champs d'expression possibles de notre désir de maîtrise, d'un point de vue sportif, économique, scientifique, etc..., alors que leur image suggère un respect absolu. 
La sensation de rupture que procure une première trace humaine dans un paysage considéré comme sauvage est à mon sens une constante intemporelle et interculturelle. Ce sont les motivations de ces gestes, les prétextes culturels qui les justifient, le jugement que nous portons sur eux et le sens que nous leur attribuons qui ont évolués. Si actuellement notre regard change, c'est peut-être que se concrétise une rupture de civilisation, et que nous l'abordons avec la nostalgie de ce que nous sommes entrain de quitter définitivement. Dés la fin du dix-neuvième siècle, les milieux artistiques considéraient que l'homme était instrumentalisé par les évolutions techniques qui le fascinent, et que celles-ci allaient créer une rupture sociale et culturelle (8). L'homme actuel continue-t-il à se servir de la technique, ou s'est-elle emparée de la société, modelant et utilisant les humains au service d'un mécanisme évolutif technocratique, sorte d'auto domestication favorisant les comportements les plus aptes à utiliser et produire de nouveaux arrangements dans la matière du monde (9) ? Car l'univers, et la vie biologique en particulier, existent parce que les états et ordonnancements de la matière sont instables et en permanente évolution. Modifier le monde qui nous entoure est donc avant tout obéissance à cette force fondatrice. Croire que notre technologie et notre culture nous arrachent à la condition dans laquelle la nature nous avait placé n’est qu'illusion, en ce sens qu'elles ne font qu'accélérer et prolonger les conséquences de lois universelles. Nous ajoutons certes notre singularité, mais notre marge de manœuvre semble finalement assez limitée : nous produisons une autre nature, qui émerge du biologique comme celui-ci a émergé du minéral.

\section{Conclusion}

Il semble que la confrontation d'un signe humain non fonctionnel et infime avec un environnement totalement " naturel " n'ait plus de sens aujourd'hui. Le paysage idéal de l'homme du vingt et unième siècle est soit la maîtrise totale symbolisée par la ville futuriste parfaite, soit l'enclave vierge de toute action humaine. A défaut de nous considérer encore comme le centre et la raison d'être de l'Univers, l'aménagement des espaces à notre porté aux besoins de notre existence s'avère beaucoup plus complexe et incertain que nous l'imaginions il y a quelques dizaines d'années encore. Incapables d'accepter les paradoxes ni de les gérer, nous supportons mal de nous voir vagabonder dans l'espace et le temps : nous voudrions en emplir l'infini, ou n'avoir jamais existé. 
(1) EDDOWES, Mark. Transformation des pratiques religieuses de la fin du culte hui arii. Bulletin de la Société des Etudes Océaniennes, Tahiti, n 290, décembre 2001.

(2) FOREST, Jean Paul. " Art rupestre contemporain " . Bulletin de la Société des Etudes Océaniennes , Tahiti, nº 303, décembre 2005.

(3) SIMONDON, Gilbert. Du mode d'existence des objets techniques. Paris : Aubier. 1989.

(4) TERRASSON, François. La peur de la nature. Paris : Sang de la Terre. 1997.

(5) OTTE, Marcel. Les origines de la pensée. Liège : Mardaga. 2001.

(6) OTTE, Marcel. La préhistoire. Bruxelles : De Boeck. 2003.

(7) CROSSMAN, Sylvie \& BAROU, Jean-Pierre. Enquête sur les savoirs indigènes. Paris : Folio - 2005.

(8) COLLECTIF. Esthétique et philosophie de l'art. Bruxelles : De Boeck. 2002

(9) ELHUL, Jacques. L'empire du non sens. Paris : P.U.F. 1980

Recebido para publicação em janeiro de 2007 Aprovado para publicação em julho de 2007 